\title{
Effect of Dietary EGCG on Normal and Vitamin E and Selenium Deficient Rats
}

\author{
Juan E. Andrade ${ }^{1^{*}}$, John R. Burgess ${ }^{2 *}$ \\ ${ }^{1}$ Department of Food Science and Human Nutrition, University of Illinois, Champaign-Urbana, USA; ${ }^{2}$ Department of Nutrition Sci- \\ ence, Purdue University, West Lafayette, USA. \\ Email: jandrade@illinois.edu, ${ }^{*}$ burgesjr@purdue.edu
}

Received April $6^{\text {th }}, 2013$; revised May $6^{\text {th }}, 2013$; accepted May $14^{\text {th }}, 2013$

Copyright (C) 2013 Juan E. Andrade, John R. Burgess. This is an open access article distributed under the Creative Commons Attribution License, which permits unrestricted use, distribution, and reproduction in any medium, provided the original work is properly cited.

\begin{abstract}
The tea catechin EGCG has been postulated to provide health benefits in humans, to some extent, as an antioxidant. The dose-response effect of dietary EGCG $(0,30,60$, or $120 \mathrm{mg} / \mathrm{kg}$ diet $)$ was tested in rats under high versus low oxidative stress conditions that were created by feeding diets adequate in vitamin E and Se (Lox), or deficient in both (Hox) for six weeks. Effects on growth, quinone reductase (NQO1) activity, $\mathrm{F}_{2}$-isoprostanes and nutrient antioxidant amounts in the liver were evaluated as markers of nutrient deficiency and oxidative status. Under Hox conditions consumption of EGCG only at the lowest dose was partially associated with a protection against oxidative stress, reflected by a delay in growth deceleration, but no protection against lipid oxidation. Elevated liver NQO1 activity was observed in this group ( $>4$-fold) increasing with the dose; but it was not associated with antioxidant protection. In contrast, under Lox conditions consumption of EGCG was associated with antioxidant activity reflected in a reduction $(>30 \%)$ in $\mathrm{F}_{2}$-isoprostanes and protection of CoQ reduced status in the liver. Overall these results suggest that the antioxidant effect of EGCG in vivo depends on the level of oxidative stress and the presence of other nutrient antioxidants.
\end{abstract}

Keywords: Vitamin E Deficiency; Oxidative Stress; EGCG; Flavonoid; Rat; Antioxidant

\section{Introduction}

Aerobic organisms are in constant interaction with molecular oxygen as part of the energy yielding processes in cells. Because this process is not perfect, this adaptation to use oxygen as the final acceptor of electrons in the process of trapping energy is translated in the accumulation of oxygen free radicals or reactive oxygen species (ROS). Their uncontrolled formation and accumulation within the body causes oxidative damage to proteins, membranes and genes leading to oxidative stress [1]. Oxidative stress is implicated in the development of many chronic diseases such as macular degeneration, cancer, cardiac disease, and premature aging; however, its exact role in a given disease is often unclear [2].

Consumption of diets rich in plant foods, such as fruits, vegetables and herbs, has been negatively correlated with the risk for a variety of chronic diseases, particularly cardiovascular disease and some types of cancer [3]. These beneficial effects have largely been attributed to their

\footnotetext{
"Corresponding author.
}

high content of dietary fiber and non-nutrient plant chemicals such as flavonoids and terpenes. Over the past years, the use of flavonoids as health promoters has become increasingly appreciated. The main stated hypothesis is that flavonoids, to some extent, mediate the beneficial effects derived from an enriched fruit and vegetable diet in part by their multiple antioxidant properties [4].

The infusion from Camelia sinensis leaves, also known as green tea, is the second most consumed beverage in the World and it is considered an excellent source of flavonoid antioxidants. Multiple epidemiological studies have linked green tea consumption with the reduction for the risk of disease conditions such as multiple cancer types and cardiovascular disease [5]. Fresh tea leaves are rich in flavanol monomers known as catechins. Epigallocatechin-3-gallate (EGCG; Figure 1) is the major catechin monomer in green tea and to which most of the green tea beneficial properties are attributed [6].

Multiple in vitro and ex vivo studies support the role of green tea polyphenols and EGCG as powerful anticancer agents, apoptosis stimulators, phase II enzyme inducers, 
<smiles>O=C(O[C@H]1Cc2c(O)cc(O)cc2O[C@H]1c1cc(O)c(O)c(O)c1)c1cc(O)c(O)c(O)c1</smiles>

Figure 1. Epigallocatechin-3-Gallate structure.

and multiradical scavenger antioxidants [7]. Most of these studies documenting the antioxidant activity of the tea flavonoids were performed in isolated situations simulating cellular in vivo conditions. Because flavonoids, like EGCG, undergo extensive metabolism by gut microflora, intestinal and liver enzymes, it is unrealistic to assume that their non-metabolized forms, frequently used at super-physiological concentrations in vitro, directly account for the observed benefits by providing antioxidant protection in vivo [8]. Consequently, it is important to understand their role as antioxidants, while considering their bioavailability and metabolism in vivo. Although the field of phytochemicals and health has grown considerably in the last decade, there is still very limited information about flavonoid antioxidant properties in vivo [4].

In the present study we have used an animal model of chronic oxidative stress - rats deficient in vitamin E and selenium - to test whether dietary EGCG can provide protection against the symptoms of antioxidant nutrient deficiency and enhance antioxidant status under normal conditions in vivo.

\section{Materials and Methods}

All the chemicals used in the experiments, unless otherwise specified, were purchased from Sigma Chemical Co. (St. Louis, MO). Stock solutions $(20 \mathrm{mg} / \mathrm{mL}$ in ethanol) were made within the week of the experiments and stored in the dark either at 4 or $-20^{\circ} \mathrm{C}$.

\subsection{Animals and Experimental Design}

Fifty (40 - 50 g) male Long-Evans Hooded rats (Harlan, Indianapolis, IN) were received after weaning and placed on one of eight experimental diets. The basal diet was made with torula yeast to control Se and the oils were tocopherol-stripped to control vitamin E. A low oxidative stress diet (Lox) was supplemented with tocopheryl acetate $(100 \mathrm{mg} / \mathrm{kg}$; MP Biomedicals, Cleveland, OH) and sodium selenite $(0.5 \mathrm{mg} / \mathrm{kg})$, and the high oxidative stress diet (Hox) was deficient in both antioxidant nutriaents as described by Kuo, et al. [9]. To assure proper mixture, pure EGCG was added to $500 \mathrm{~g}$ of the sugar and used at increasing concentrations: $0,30,60$ and $120 \mathrm{mg} / \mathrm{kg}$ of diet. Then, individual mixtures were added to the remaining dry ingredients according to the diet composition. These catechin amounts are approximately equivalent to consuming 0, 1.6, 3.2 and $6.4 \mathrm{mg}$ EGCG/ $\mathrm{kg}$ body weight per day by the end of the study. These amounts are within the range a US individual $(70 \mathrm{~kg})$ would consume from 1, 2 and 4 cups $(240 \mathrm{~mL})$ of green tea a day, if consuming from common US vendors of green tea products supplying on average $55.5 \mathrm{mg}$ EGCG per 100 $\mathrm{mL}$ of prepared tea [10]. The animals were housed individually and maintained at $24^{\circ} \mathrm{C}$ with $12 \mathrm{~h}$ light-dark cycles. Rats had free access to food and deionized water at all times and were fed with diets for six weeks. For food intake compliance, food consumption and spillage was measured every other day using a small electronic scale. Body weight was measured weekly throughout the study. At the end of the study, animals were fasted for 12 $\mathrm{h}$ before an intraperitoneal injection of ketamine (90 $\mathrm{mg} / \mathrm{kg}$ ) and xylazine $(10 \mathrm{mg} / \mathrm{kg})$ and euthanized by exsanguination. Plasma was immediately collected and liver and other tissues were perfused with physiological saline, removed, weighed, snap-frozen in liquid nitrogen, and stored at $-80^{\circ} \mathrm{C}$ until further analysis. Purdue University Animal Care and Use Committee approved all the experimental procedures.

\subsection{Tissue Preparation}

Representative liver samples were thawed on ice, weighed, and homogenized $(20 \%, \mathrm{w} / \mathrm{v})$ in ice cold $50 \mathrm{mM}$ Tris$\mathrm{HCl}$ buffer $\mathrm{pH} 7.4$ containing $150 \mathrm{mM}$ potassium chloride, $1 \mathrm{mM}$ sodium EDTA and $0.1 \%$ BHT (except for NQO1 aliquots) for analysis of fat-soluble antioxidants, quinone reductase (NQO1) activity, and $\mathrm{F}_{2}$-isoprostanes (type A homogenates); or in ice cold 15\% perchloric acid containing $1 \mathrm{mM}$ EDTA for water-soluble antioxidants determination (type B homogenates). The two types of liver homogenates were made on ice using a PotterElvehjem apparatus attached to a rotatory device. After six strokes at medium-high speed, type A liver homogenates were centrifuged at $1000 \times \mathrm{g}$ for 15 minutes at $4^{\circ} \mathrm{C}$. Supernatants were immediately transferred, vortexed and aliquoted into amber vials, and kept frozen at $-80^{\circ} \mathrm{C}$ until further analysis. Upon protein precipitation type B homogenates were centrifuged at $1000 \times \mathrm{g}$ for 15 minutes at $4^{\circ} \mathrm{C}$. Clear supernatants were immediately transferred, vortexed and aliquoted into small amber vials, snapfrozen in liquid nitrogen, and stored at $-80^{\circ} \mathrm{C}$ until further analysis.

\subsection{Analyses}

\subsubsection{Determination of Fat-Soluble Antioxidants}

Extraction of tocopherols and ubiquinones from type A 
homogenates was done as described by McCrehan [11] with minor changes. Briefly, a ratio of $2: 1$ ethanol to tissue homogenates was used and spiked with $10 \mu \mathrm{L}$ of 100 $\mu \mathrm{M}$ of $\delta$-tocopherol as internal standard. Fat-soluble antioxidants were extracted using hexane. The upper hexane layer was evaporated under nitrogen. After resuspension in ethanol samples were filtered using $0.22 \mu \mathrm{m}$ nylon syringe filters (Osmonics Inc.; Minnetonka, MN) directly into HPLC vials.

Simultaneous separation and quantification of $\alpha$-tocopherol, $\gamma$-tocopherol, $\delta$-tocopherol, and $\mathrm{CoQ}_{9}$ and $\mathrm{CoQ}_{10}$ reduced and oxidized forms was carried out by HPLC reverse phase with electrochemical detection using the CoulArray $^{\circledR}$ (Model 5600A) with a binary pump system (ESA, Inc., Chelmsford, MA). The column MD-150 column $(\mathrm{C} 18,150 \times 3.2 \mathrm{~mm}$ id, $3 \mu \mathrm{m}$ particle size; ESA, Inc., Chelmsford, MA) was equilibrated in mobile phase A, methanol: $0.02 \mathrm{M}$ Acetate buffer $\mathrm{pH} 4.0(90: 10 \mathrm{v} / \mathrm{v})$. Fat soluble antioxidants were gradient-eluted with mobile phase $\mathrm{B}$, methanol: n-propanol: $0.1 \mathrm{M}$ acetate buffer $\mathrm{pH} 4.0(78: 20: 2 \mathrm{v} / \mathrm{v})$. Monitoring of antioxidants was carried out with an electrochemical detector as described in CoulArray Applications [12]. Eluted compounds were quantified using HPLC grade external standards. Results were expressed per mg of protein.

\subsubsection{Determination of Water-Soluble Antioxidants}

Simultaneous determination of ascorbate and glutathione (GSH) from type B homogenates was adapted from Stempak, et al. [13] and Kristal, et al. [14]. Briefly, type $\mathrm{B}$ homogenates were diluted with double deionized water and filtered directly into HPLC vials with $0.45 \mu \mathrm{m}$ RCmembrane filters (Ministart RC 4; Sartorius AG, Göttingen, Germany).

Separation and quantification of liver water-soluble antioxidants was carried out by HPLC reverse phase with electrochemical detection using the CoulArray ${ }^{\circledR}$ (Model 5600A) with a binary pump system (ESA, Inc., Chelmsford, MA). The column Supelcosil LC-18-T-150 (150 × $4.6 \mathrm{~mm}$ id, $3 \mu \mathrm{m}$ particle size; Supelco-Sigma, St Louis, MO) was equilibrated in mobile phase A, $50 \mathrm{mM}$ sodium phosphate buffer $\mathrm{pH}$ 3.0. Water-soluble antioxidants were gradient-eluted with mobile phase $\mathrm{B}$, acetonitrile: $50 \mathrm{mM}$ sodium phosphate buffer $\mathrm{pH}$ 3.0: methanol (50:30:20 $\mathrm{v} / \mathrm{v})$. Detection was carried out using the 8-channel CoulArray ${ }^{\circledR}$ electrochemical system. Eluted compounds were quantified using HPLC grade external standards. Results were expressed in grams of fresh liver.

\subsubsection{Total $F_{2}$-Isoprostane Analysis}

Analysis of liver total (free and esterified) $\mathrm{F}_{2}$-isoprostanes (8-epi $\mathrm{PGF}_{2} \alpha$ ) as markers of oxidative stress was carried out using type A liver homogenates and a ELISA kit from Cayman Chemical (Cayman Chemical, Ann Ar- bor, MI). Previous to analysis, samples were subjected to a purification step using an 8-epi $\mathrm{PGF}_{2} \alpha$ affinity column kit and treated as indicated by the manufacturer. Results were expressed per mg of protein.

\subsubsection{Quinone Reductase Activity}

Determination of $\mathrm{NAD}(\mathrm{P}) \mathrm{H}$ :quinone oxido-reductase (NQO1, EC 1.6.99.2) activity as a marker of oxidative stress and adaptation was measured using type A liver homogenates and a plate reader (PowerWaveX, BIOTEK Instruments, Inc., Winooski, VT) as previously described by Prochaska and Santamaria [15].

\subsubsection{Protein Concentration}

Protein was determined by the bicinchoninic acid method of Smith, et al. [16] using type A liver homogenates. All results, unless otherwise stated within the text, are expressed per mg of total protein.

\subsection{Statistical Analyses}

Data are shown as the means \pm SEM of 6 - 8 measurements/group. Either two-way ANOVA or one-way ANOVA was used to analyze two or one factor effects at $P<0.05$ using the SAS software (SAS Institute, Cary, NC). Variances were isolated and analyzed using the F-test $(P<$ 0.05 ) for the main effects of oxidative stress, Lox vs. Hox, dose effects of selected EGCG concentrations and main effects interactions. Data were subjected to logarithmic transformation where necessary to achieve homogeneity of variances. Proper analysis of statistical assumptions such as homogeneity of variances, and uniformity of residuals, for one-way ANOVA and two-way ANOVA were also performed. If differences were observed, post-hoc analysis of multiple mean comparisons within each oxidative condition and between each treatment against Lox and Hox controls was performed using least square difference with Scheffe correction. Differences were considered significant when $P<0.05$. Repeated measures ANOVA was used to determine differences in animal total weight, weight gain and food consumption among groups. Growth rate was calculated as the slope between time in weeks and weight in grams. Slope was calculated in Excel and a linear model for growth was assumed, based on the best fitted model for growth using the Lox control group data. Pearson correlation coefficient was calculated to establish the strength and association of all outcome variables.

\section{Results}

\subsection{Effects of Oxidative Condition and Dietary EGCG on Growth and Food Intake}

Consumption of the nutrient deficient diets led to stunted growth and to hepatomegalia compared to control ani- 
mals fed diets sufficient in vitamin $\mathrm{E}$ and selenium. Final body weights, weight gain and growth rates were significantly lower in Hox rats consuming the control diet than in those fed the Lox control diet (Table 1; $P<0.05$ ). Longitudinal analysis demonstrated that changes in body weight were apparent and significant after the fifth week of experimentation (Figure 2). Supplementation with EGCG at the lowest and highest dose prevented growth deceleration in Hox animals and resulted in intermediate values that were not different from both controls. On the other hand, dietary EGCG, at any dose, did not affect final total weights, total weight gain and growth rates in animals under normal conditions (Figure 2, Table 1). Effects on growth were independent of food consumption. Food intake during the six weeks of the study and for all the groups was on average $\sim 13.2 \mathrm{~g} / \mathrm{rat} /$ day (Table 1). Antioxidant nutrient deficient rats showed higher liver weights compared to their counterparts, and this physiological response to oxidative stress was neither reduced nor enhanced with EGCG supplementation (Table 1).

Table 1. Effect of oxidative stress condition and dietary EGCG on animal performance, food intake, and liver fat- and watersoluble antioxidants.

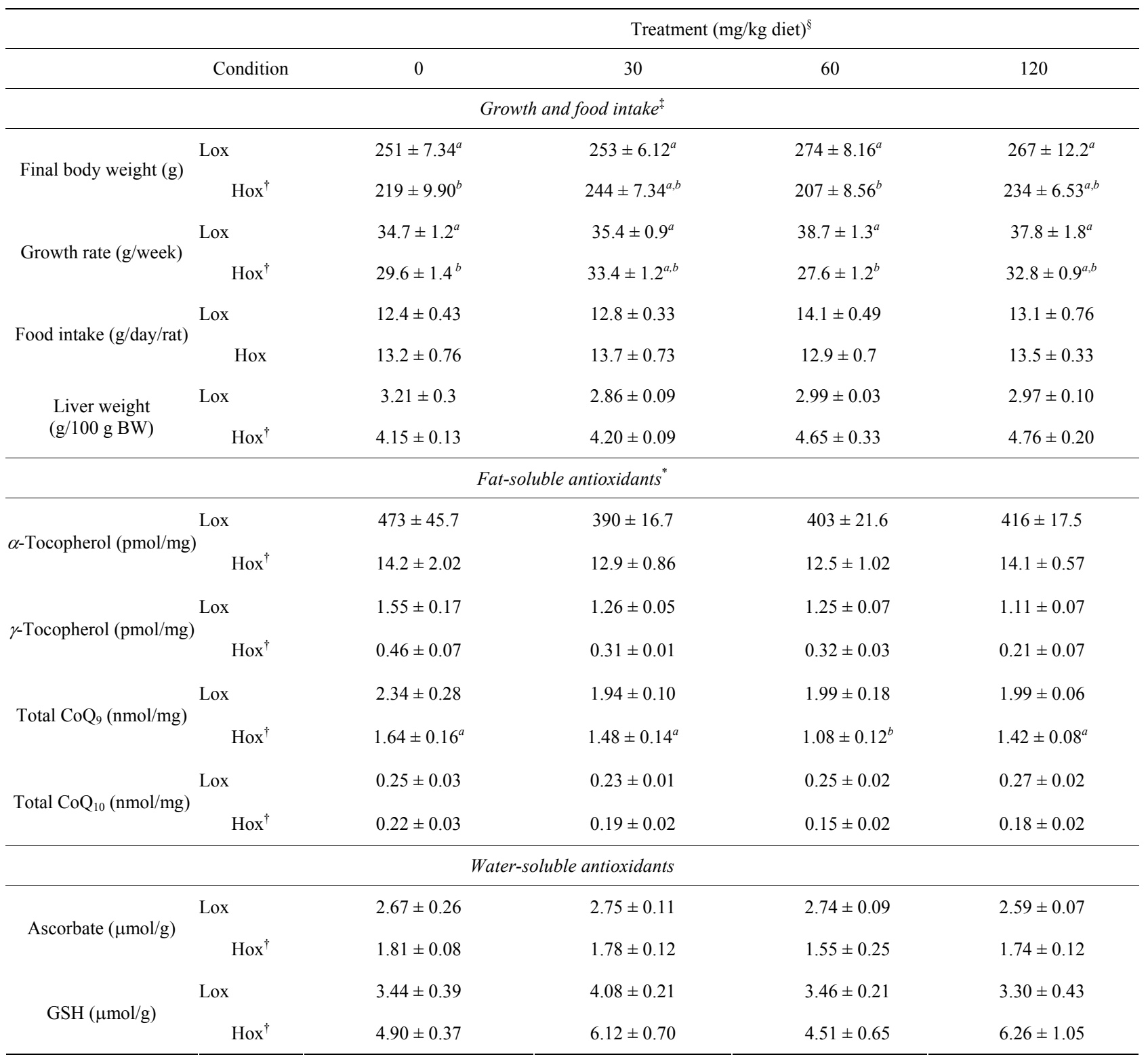

$\overline{{ }^{\S} \text { Values are arithmetic means } \pm \mathrm{SEM} ; \mathrm{n}=6-8 \text { for each treatment group. }{ }^{\dagger} \text { Represents statistical differences between oxidative conditions after two-way }}$ ANOVA, $P<0.05$. ${ }^{\ddagger}$ For growth and food intake variables and water-soluble antioxidant (unit per $g$ of liver), letters between each treatment group and the controls represent statistical differences after one-way ANOVA and post-hoc analysis between each group and both controls (LSD; $P<0.05$ ). For fat-soluble antioxidants (unit per mg of protein), letters between each treatment group and the controls represent statistical differences after one-way ANOVA and post-hoc analysis among treatments within each condition (LSD; $P<0.05$ ). 


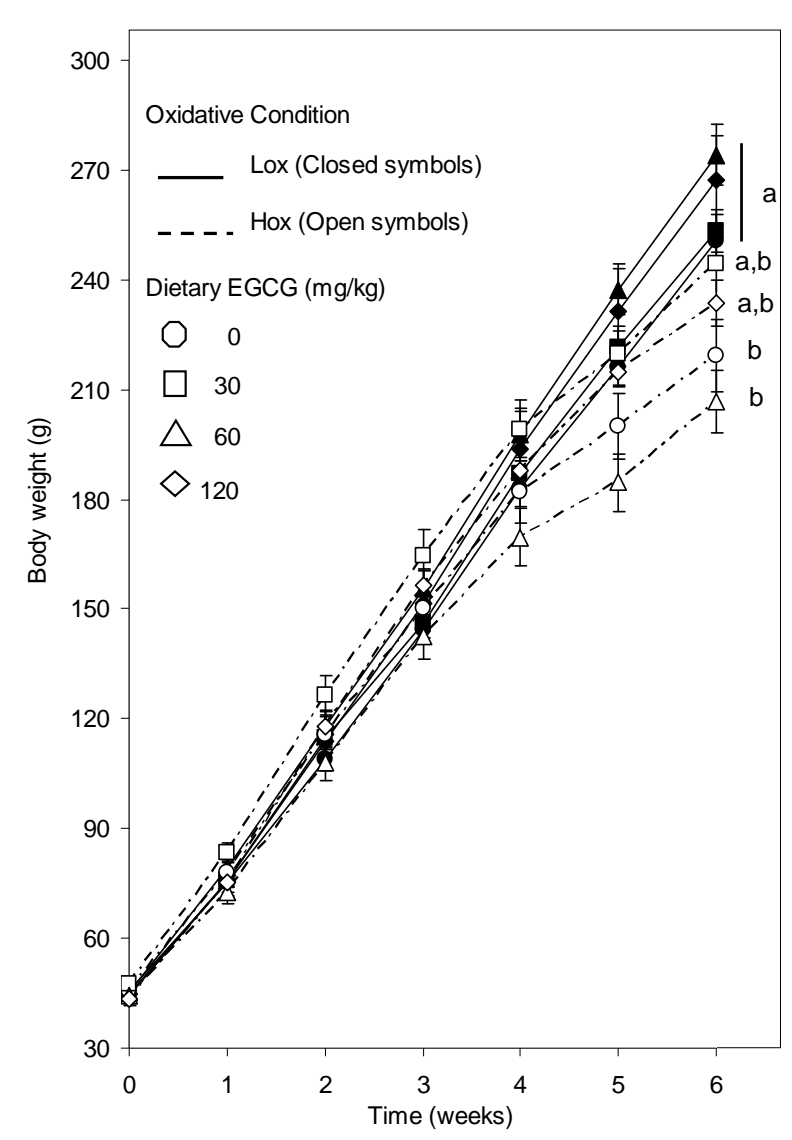

Figure 2. Effect of oxidative stress condition and dietary EGCG on rat growth during six weeks. Each data point represents the arithmetic mean \pm SEM. Different (a,b) superscripts represent statistical differences among multiple comparisons after Repeated Measures ANOVA (Time $x$ Oxidative Condition x EGCG, $P<0.05$ ) conducted for each time interval. Changes in total body weight were significant at the fifth week, but for clarity superscripts are not included.

\subsection{Effects of Oxidative Condition and Dietary EGCG on Liver Fat-Soluble Antioxidants}

As expected, $\alpha$ - and $\gamma$-tocopherol levels were significantly lower in nutrient deficient animals than in their counterparts (Table 1; $P<0.01$ ). Dietary EGCG did not affect liver $\alpha$ - and $\gamma$-tocopherol concentrations in either condition (Table 1).

The oxidative condition and dietary EGCG affected liver $\mathrm{CoQ}$ status. Total $\mathrm{CoQ}_{9}$ and $\mathrm{CoQ}_{10}$ amounts were reduced as a result of the nutrient deficient diet (Table 1) and EGCG supplementation did not prevent this effect. Hox rats consuming EGCG at $60 \mathrm{mg} / \mathrm{kg}$ of diet showed even lower liver total amounts of both CoQ homologs than the control group $(P<0.05)$, but without a clear trend at a higher dose. Animals under Lox conditions consuming EGCG showed intermediate amounts of liver total $\mathrm{CoQ}_{9}$, but not $\mathrm{CoQ}_{10}$ compared to both controls
(Table 1). CoQ RED/OX ratio is a representation of the reduced state of ubiquinol against its oxidized form ubiquinone. Hox rats showed higher liver CoQ RED/OX ratios for both CoQ forms compared to their Lox counterparts (Figures 3(a) and (b); $P<0.05$ ). EGCG supplementation led to higher $\mathrm{CoQ}_{9}$ and $\mathrm{CoQ}_{10} \mathrm{RED} / \mathrm{OX}$ ratios in rats under Lox, but not Hox conditions (Figures 3(a) and (b); $P<0.05$ ). RED/OX ratios where significantly $(P<0.05)$ inversely associated with their total Q homologs; Pearson correlation coefficient for $\mathrm{Q}_{9}$ and $\mathrm{Q}_{10}, \rho=$ -0.52 and -0.30 , respectively. Changes in RED/OX ratios and total ubiquinone amounts were parallel between CoQ homologs.

\subsection{Effects of Oxidative Condition and Dietary EGCG on Liver Water-Soluble Antioxidants}

Animals consuming nutrient deficient diets showed lower liver ascorbate concentrations $(\sim 37 \%)$. This reduction was not prevented at any dietary EGCG level. Similarly, EGCG supplementation did not affect liver ascorbate concentrations in Lox rats (Table 1). After standardization of the variances, liver GSH concentrations were elevated in rats consuming nutrient deficient diets compared to their Lox counterparts $(P<0.05)$ and changes were not affected by dietary EGCG.

\subsection{Effects of Oxidative Condition and Dietary EGCG on Markers of Lipid Oxidation}

Liver $\mathrm{F}_{2}$-isoprostanes were determined to assess the effects of vitamin $\mathrm{E}$ and Se deficiency on the magnitude of oxidative stress represented as oxidative lipid damage. $\mathrm{F}_{2}$-isoprostanes concentrations were higher $(\sim 24 \%)$ in the liver of rats under Hox conditions compared to the controls (Figure 4; $P<0.05$ ). Rats fed the nutrient deficient diet and EGCG, at 30 and 60 , but not $120 \mathrm{mg} / \mathrm{kg}$, showed amounts of hepatic $\mathrm{F}_{2}$-isoprostanes that were not different from both control groups (after one-way ANOVA and LDS, not specified in Figure 4; $\mathrm{P}>0.05$ ). In contrast, dietary EGCG lowered the amount of $\mathrm{F}_{2}$-isoprostanes $(\sim 30 \%)$ in the liver of rats under Lox conditions (Figure 4; $P<0.05)$.

\subsection{Effects of Oxidative Condition and Dietary EGCG on Hepatic NQO1 Activity}

Consumption of the nutrient deficient diet led to more than 4-fold increase in hepatic NQO1 activity (Figure 5; $P<0.01$ ). Addition of increasing amounts of EGCG to the nutrient deficient diets led to an unexpected pattern in this enzyme activity as depicted in Figure 5. First, animals consuming nutrient deficient diets and EGCG at 30 $\mathrm{mg} / \mathrm{kg}$ had lower hepatic NQO1 activities $(P<0.05)$. This activity remained unchanged in rats in the EGCG 60 


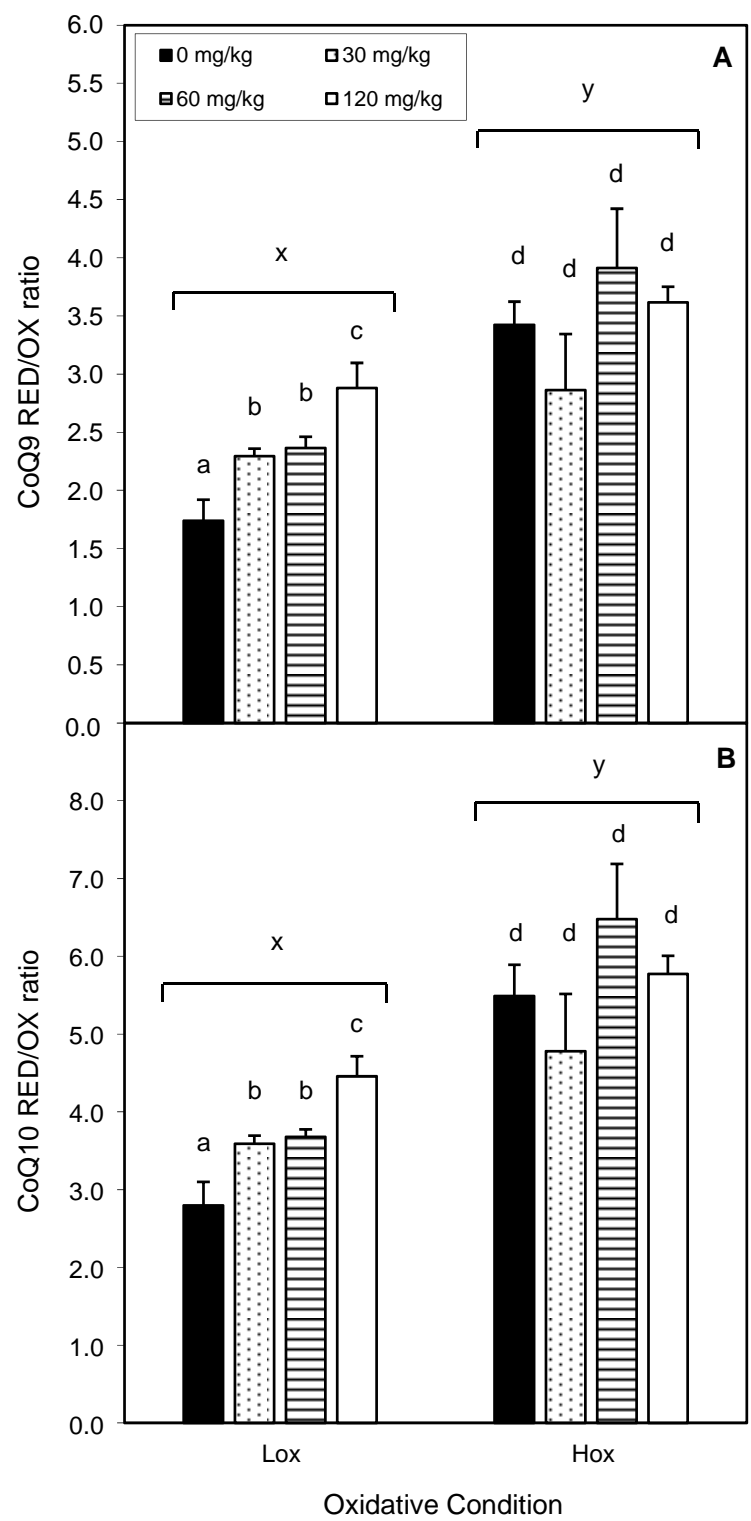

Figure 3. Effect of oxidative stress condition and dietary EGCG on hepatic RED/OX ubiquinol:ubiquinone ratio. Each bar represents the arithmetic mean \pm SEM. Different $(x, y)$ superscripts represent statistical differences between oxidative conditions after two-way ANOVA, $P<0.05$. Different $(a, b)$ superscripts represent statistical differences among multiple comparisons within each oxidative condition after one-way ANOVA and post-hoc analysis (LSD, $P<$ 0.05).

$\mathrm{mg} / \mathrm{kg}$ group, whereas it was higher in the EGCG 120 $\mathrm{mg} / \mathrm{kg}$ group $(P<0.05)$ against the Hox control group. In contrast, EGCG did not influence hepatic NQO1 activity in animals consuming the nutrient sufficient diet.

\section{Discussion}

We have found that EGCG can exhibit antioxidant activities in vivo, and that this property depends on the

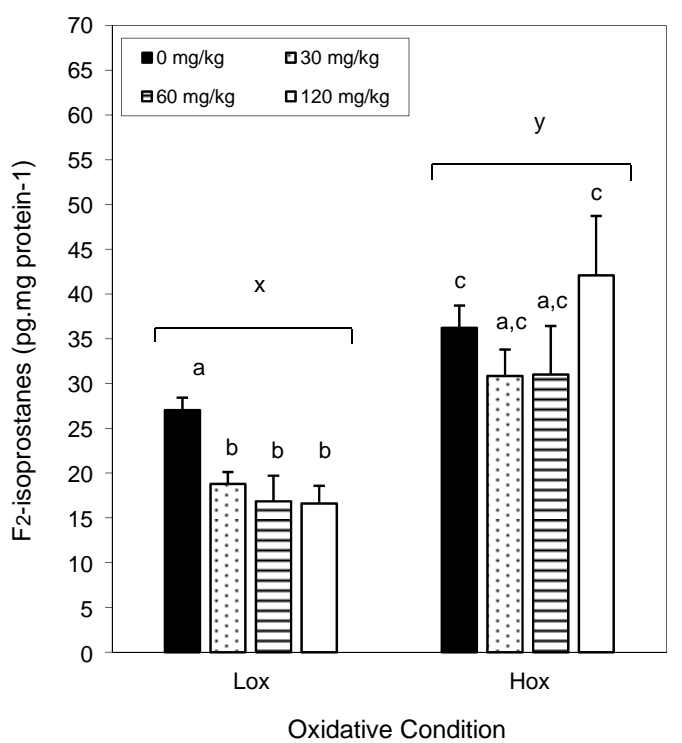

Figure 4. Effect of oxidative stress condition and dietary EGCG on hepatic $F_{2}$-isoprostanes. Each bar represents the arithmetic mean \pm SEM. Different $(x, y)$ superscripts represent statistical differences between oxidative conditions after two-way ANOVA, $P<\mathbf{0 . 0 5}$. Different (a,b) superscripts represent statistical differences among multiple comparisons within each oxidative condition after one-way ANOVA and post-hoc analysis (LSD, $P<0.05)$.

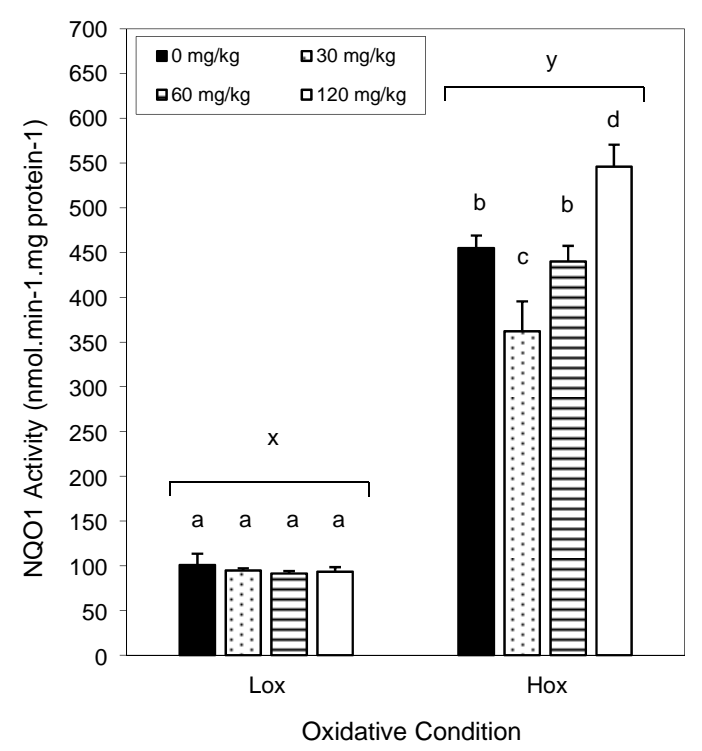

Figure 5. Effect of oxidative stress condition and dietary EGCG on hepatic NQO1 activity Each bar represents the arithmetic mean \pm SEM. Different $(x, y)$ superscripts represent statistical differences between oxidative conditions after two-way ANOVA, $P<0.05$. Different $(a, b)$ superscripts represent statistical differences among multiple comparisons within each oxidative condition after one-way ANOVA and post-hoc analysis (LSD, $P<\mathbf{0 . 0 5}$ ).

animal's oxidative stress condition. Deficiency of both vitamin $\mathrm{E}$ and selenium led to lower total body weight 
and growth rate and to hepatomegalia as previously reported [17-19]. Growth deceleration observed is mainly due to selenium deficiency as reported earlier [20]. Under similar conditions, consumption of EGCG both at the lowest and highest dose delayed growth deceleration; however, this slight protection was not strong enough to prevent liver hypertrophy. Addition of dietary EGCG did not affect animal performance or food consumption. To associate the effect on growth with protection against oxidative stress, we determined the concentrations of $\mathrm{F}_{2}$ isoprostanes and nutrient antioxidants such as vitamin $\mathrm{E}$, coenzyme Q (CoQ), ascorbate and glutathione (GSH) in the liver.

Consumption of EGCG diets led to lower hepatic lipid peroxidation (i.e., $\mathrm{F}_{2}$-isoprostanes) only in animals under normal conditions. Although there was a trend to lower lipid peroxidation in Hox animal at lower EGCG doses, this was not significant. Willcox and colleagues [21] using the vitamin $\mathrm{E}$ (not Se) deficiency model to test the antioxidant effects of quercetin, catechin, epicatechin, cocoa powder or lignin in vivo failed to observe parallel results either in normal or nutrient deficiency conditions. Despite the elevated doses used ( $>200 \mathrm{mg} / \mathrm{kg} \mathrm{bw} /$ day), these polyphenols did not prevent the accumulation of $\mathrm{F}_{2}$-isoprostanes in rats heart and plasma. Discrepancies are possibly due to the antioxidant quality of the flavonoids tested; EGCG has superior antioxidant properties in vitro when compared to other flavonoids [22].

Determination of fat-soluble, but not water-soluble antioxidants was instrumental to explain changes in $\mathrm{F}_{2}$-isoprostanes accumulation in the liver of rats under Lox, but not Hox conditions. As expected, rats under Hox conditions showed lower liver $\alpha$ - and $\gamma$-tocopherol concentrations. Choi et al. [23] reported protective effects $(\sim 10 \%$ $30 \%$ ) on serum and hepatic $\alpha$-tocopherol in rats consuming vitamin $\mathrm{E}$ (not $\mathrm{Se}$ ) deficient diets along with quercetin ( 8 and $80 \mathrm{mg} / \mathrm{kg}$ bw/day) for 4 weeks. Discrepancies with this study could be due to the level of oxidative stress provoked in the double nutrient deficiency model and the higher doses used.

Animals consuming nutrient deficient diet exhibited reduced amounts of total hepatic CoQ, but higher CoQ reduced/oxidized (ubiquinol/ubiquinone; RED/OX) ratios. Dietary EGCG neither affected the amounts of $\alpha$ and $\gamma$-tocopherol nor prevented the reduction in total CoQ concentrations or modified CoQ RED/OX ratio in rats under oxidative stress. Higher RED/OX ratios along with lower total CoQ amounts have been reported in rat testis under oxidative stress conditions caused by iron overload [24]. A higher CoQ RED/OX ratio appears to be critical in maintaining mitochondrial function as suggested by Miles et al. [25] and is inversely correlated with total liver CoQ content as our results and others have shown [26]. A higher RED/OX ratio could be ex- plained by the enhanced activity of ubiquinone reducing enzymes such as cytochrome b5 reductase and NQO1 as an adaptation to oxidative stress $[18,27]$.

In contrast to our observations in animals under Hox conditions, EGCG consumption led to higher $\mathrm{CoQ}_{9}$ and $\mathrm{CoQ}_{10} \mathrm{RED} / \mathrm{OX}$ ratios in the liver of rats under normal conditions, which represents a shift towards the reduced state along with antioxidant protection [28]. Although ubiquinol exhibits synergistic effects with vitamin E by regenerating the active form from the tocopheroxyl radical [29], the improvement in CoQ status and reduction of $\mathrm{F}_{2}$-isoprostanes was not associated with significant changes in hepatic tocopherols or water-soluble antioxidants. Liver ascorbate and GSH concentrations were increased and decreased, respectively, due to Hox conditions and changes were independent of EGCG consumption. GSH levels has been shown to increase during Hox conditions due adaptations to oxidative stress [18], conditions which leads to increased synthesis as previously reported [30]. This implies that albeit its partial hydrophilic properties, EGCG may interact better with fat-soluble compounds in vivo. Tocopherol sparing effects of dietary flavonoids have been observed in different animal studies under normal conditions $[31,32]$ or after consumption of high fat diets $[33,34]$. It is possible that these sparing effects were due to the high doses used in these studies, where the dietary amounts of single flavonoids or their mixtures (green tea) where larger than in our study, $8-500 \mathrm{mg} / \mathrm{kg}$ bw/day. Nonetheless, these studies did not assess the status of CoQ in plasma or tissues and its potential role regenerating vitamin E.

Multiple quinone oxido-reductases are involved in the reduction of oxidized CoQ to support its role as an antioxidant. NQO1 is cytosolic flavoenzyme that catalyzes the two-electron reduction of exogenous quinones and nutrients, such as ubiquinone and vitamin E quinone [35]. NQO1 also plays a role in the adaptation process to oxidative stress in mammals as described earlier [18,27,36]. The nqo 1 gene is regulated through cis-coding sequences at the proximity of the promoter region known as the electrophile-responsive element (EpRE) and the xenobiotic response element (XRE) [37]. Induction of nqo1 has been demonstrated to protect against the cytotoxicity, mutagenicity and carcinogenicity of many compounds and has been associated with the potential protective role of plant chemicals, including flavonoids [38,39]. In this study, dietary EGCG led to increased hepatic NQO1 activity in animals within the Hox, but not the Lox condition. Different reports have demonstrated that EGCG induces nqo1 through both EpRE [40] and XRE promoters [41]. Moreover, Katiyar et al. [42] and Khan et al. [43] demonstrated that consumption of tea catechins induced phase II enzymes, including NQO1, in various tissues of mice. In contrast to these reports, we did not observe any 
changes in hepatic NQO1 activity in rats under Lox conditions at any dose used. It is possible that those results could be explained by the synergistic effects of the extract containing multiple tea catechins $[44,45]$ along with the elevated doses used $(0.82 \mathrm{mg}$ EGCG $/ \mathrm{mL}$ drinking water). Thus, our results do not support involvement of NQO1 eliciting the shift in CoQ towards its reduced state under Lox conditions. This shift might have occurred due to a direct or indirect interaction with the ubiquinol/tocopherol regeneration system in the lipid compartment [46,47].

The synergistic interaction between vitamin $\mathrm{E}$ and EGCG in vitro has been reported [48-51]. Thus, we propose that EGCG indirectly spared the oxidation of ubiquinol, resulting in higher RED/OX ratios, by scavenging oxygen and other type of radicals [52,53] directly, or by regenerating tocopherol in the lipid compartment; which resulted in lower formation of lipid peroxidation products. In this study we used a higher dose of dietary vitamin $\mathrm{E}$ $(100 \mathrm{mg} / \mathrm{kg})$ to elicit clear differences between oxidative conditions. As the amounts of tocopherol were significantly higher, finding a small difference due to EGCG consumption would have required higher power and significantly lower amounts of the vitamin such as it was reporter earlier $(\sim 20 \mathrm{mg} / \mathrm{kg})[31,32]$.

Similarly as we reported earlier [18], nutrient deficient animals showed higher ( $>4$-fold) hepatic NQO1 activities. EGCG consumption led to even higher hepatic NQO1 activities in nutrient deficient animals only, but this effect was neither linear nor associated with antioxidant protection. Hepatic NQO1 activity was reduced (20\%) in animals receiving the lowest dose only, while in those consuming higher EGCG doses showed higher rather than lower NQO1 activities. If EGCG was an antioxidant, we were expecting protection reflected in lower rather than higher activities. We found similar trend in hepatic NQO1 activities under Hox conditions when evaluating the antioxidant properties of the citrus flavanone naringenin [19]. It is possible that oxidative stress conditions influenced the metabolism of naringenin and EGCG, both flavonoids with similar carbon backbone, resulting in related oxidized, quinone containing metabolites with differing potency toward induction of $n q o 1$. Muzolf-Panek et al. [54] demonstrated that oxidized quinone metabolites of EGCG were able to induce $n q o 1$ gene higher than the parent compound using Hepa1c1c7 mouse hepatoma cells stably transfected with a luciferase reporter gene. Moreover, this group showed the EpRE-mediated response to catechins was modulated under oxidative stress, with higher induction in cells with reduced GSH levels and vice versa. In our study, it is expected that most EGCG was metabolized even before the aglycone form reaches the liver, which could reduce its bioactivity. Nonetheless, current evidence also has shown that oxidative stress leads to higher expression and activity of beta-glucuronidase [55], and enzyme capable to free-up aglycone metabolites of flavonoids from glucuronic acid. Thus, the accumulation of EGCG quinone metabolites under Hox conditions could explain higher hepatic NQO1 activities after increased intake of EGCG.

\section{Conclusion}

A low dose of EGCG was loosely associated with antioxidant protection in animals under Hox conditions; supported by the delay in growth deceleration and lower function of NQO1 in the liver, but not from accumulation of lipid peroxidation products or antioxidant molecules. Consumption of higher amounts of dietary EGCG did not provide additional antioxidant protection and this was only associated with higher NQO1 activities in the liver. Increased NQO1 activity is suggested to occur as a result of EGCG metabolites formed under Hox conditions, with better capacity to induce the NQO1 gene. In addition, EGCG elicited antioxidant activities in vivo with sufficient amounts of vitamin $\mathrm{E}$ and Se. Consumption of EGCG led to a reduction in the amounts of liver $\mathrm{F}_{2}$-isoprostanes, along with higher CoQ RED/OX ratio, possibly as a result of interaction with the ubiquinol/tocopherol regeneration system. In conclusion, EGCG exhibits antioxidant properties in vivo only in the presence of sufficient amounts of nutrient antioxidants. There is no consistent evidence that EGCG provides antioxidant protection against the oxidative stress symptoms derived from vitamin $\mathrm{E}$ and Se deficiency. Further studies should be directed at exploring the absorption and relationship of nutrient antioxidants and flavonoids in vivo under different conditions of oxidative stress.

\section{Acknowledgements}

This work was supported by The Botanicals Center for Age Related Diseases at Purdue University, West Lafayette, IN.

\section{REFERENCES}

[1] J. M. Gutteridge and B. Halliwell, "Free Radicals and Antioxidants in the Year 2000. A Historical Look to the Future," Annals of the New York Academy of Sciences, Vol. 899, 2000, pp. 136-147. doi:10.1111/j.1749-6632.2000.tb06182.x

[2] B. Halliwell, "Antioxidants in Human Health and Disease," Annual Review of Nutrition, Vol. 16, 1996, pp. 3350. doi:10.1146/annurev.nu.16.070196.000341

[3] P. M. Kris-Etherton, K. D. Hecker, A. Bonanome, S. M. Coval, A. E. Binkoski, K. F. Hilpert, A. E. Griel and T. D. Etherton, "Bioactive Compounds in Foods: Their Role in the Prevention of Cardiovascular Disease and Cancer," American Journal of Medicine, Vol. 113, No. S9B, 2002, 
pp. 71S-88S. doi:10.1016/S0002-9343(01)00995-0

[4] C. Rice-Evans, "Flavonoid Antioxidants," Current Medicinal Chemistry, Vol. 8, No. 7, 2001, pp. 797-807. doi:10.2174/0929867013373011

[5] V. Crespy and G. Williamson, "A Review of the Health Effects of Green Tea Catechins in in Vivo Animal Models," Journal of Nutrition, Vol. 134, No. 12S, 2004, pp. 3431S-3440S.

[6] J. V. Higdon and B. Frei, "Tea Catechins and Polyphenols: Health Effects, Metabolism, and Antioxidant Functions," Critical Reviews in Food Science and Nutrition, Vol. 43, No. 1, 2003, pp. 89-143. doi:10.1080/10408690390826464

[7] C. S. Yang, P. Maliakal and X. Meng, "Inhibition of Carcinogenesis by Tea," Annual Review of Pharmacology and Toxicology, Vol. 42, 2002, pp. 25-54. doi:10.1146/annurev.pharmtox.42.082101.154309

[8] H. H. Chow, Y. Cai, I. A. Hakim, J. A. Crowell, F. Shahi, C. A. Brooks, R. T. Dorr, Y. Hara and D. S. Alberts, "Pharmacokinetics and Safety of Green Tea Polyphenols after Multiple-Dose Administration of Epigallocatechin Gallate and Polyphenon E in Healthy Individuals," Clinical Cancer Research, Vol. 9, No. 9, 2003, pp. 3312-3319.

[9] C. F. Kuo, S. Cheng and J. R. Burgess, "Deficiency of Vitamin E and Selenium Enhances Calcium-Independent Phospholipase A2 Activity in Rat Lung and Liver," Journal of Nutrition, Vol. 125, No. 6, 1995, pp. 1419-1429.

[10] S. M. Henning, C. Fajardo-Lira, H. W. Lee, A. A. Youssefian, V. L. Go and D. Heber, "Catechin Content of 18 Teas and a Green Tea Extract Supplement Correlates with the Antioxidant Capacity," Nutrition and Cancer, Vol. 45, No. 2, 2003, pp. 226-235. doi:10.1207/S15327914NC4502 13

[11] W. A. McCrehan, "Determination of Retinal, a-Tocopherol, and b-Carotene in Serum by Liquid Chromatography," Methods in Enzymology, Vol. 189, 1990, pp. 172-181. doi:10.1016/0076-6879(90)89288-S

[12] ESA, "Simultaneous Analysis of Carotenoids, Retinoids, Tocopherols, Vitamin K1 and Coenzyme Q10 in Plasma," 1996.

[13] D. Stempak, S. Dallas, J. Klein, R. Bendayan, G. Koren and S. Baruchel, "Glutathione Stability in Whole Blood: Effects of Various Deproteinizing Acids," Therapeutic Drug Monitoring, Vol. 23, No. 5, 2001, pp. 542-549. doi:10.1097/00007691-200110000-00008

[14] B. S. Kristal, K. E. Vigneau-Callahan and W. R. Matson, "Simultaneous Analysis of the Majority of Low-Molecular-Weight, Redox-Active Compounds from Mitochondria," Analytical Biochemistry, Vol. 263, No. 1, 1998, pp. 18-25. doi:10.1006/abio.1998.2831

[15] H. Prochaska and A. B. Santamaria, "Direct Measurement of NAD(P)H: Quinone Reductase from Cells Cultured in Microtiter Wells: A Screening Assay for Anticarcinogenic Enzyme Inducers," Analytical Biochemistry, Vol. 169, No. 2, 1998, pp. 328-336. doi:10.1016/0003-2697(88)90292-8

[16] P. K. Smith, R. I. Krohn, G. T. Hermanson, A. K. Mallia, F. H. Gartner, M. D. Provenzano, E. K. Fujimoto, N. M.
Goeke, B. J. Olson and D. C. Klenk, "Measurement of Protein Using Bicinchoninic Acid," Analytical Biochemistry, Vol. 150, No. 1, 1985, pp. 76-85. doi:10.1016/0003-2697(85)90442-7

[17] J. R. Burgess and C. F. Kuo, "Increased Calcium-Independent Phospholipase A2 Activity in Vitamin E and Selenium-Deficient Rat Lung, Liver and Spleen Cytosol Is Time-Dependent and Reversible," Journal of Nutritional Biochemistry, Vol. 7, No. 7, 1996, pp. 366-374. doi:10.1016/S0955-2863(96)00057-5

[18] R. D. Cabo, J. R. Burgess and P. Navas, "Adaptations to Oxidative Stress Induced by Vitamin E Deficiency in Rat Liver," Journal of Bioenergetics and Biomembranes, Vol. 38, No. 5-6, 2006, pp. 309-317. doi:10.1007/s10863-006-9050-1

[19] J. E. Andrade and J. R. Burgess, "Effect of the Citrus Flavanone Naringenin on Oxidative Stress in Rats," Journal of Agricultural and Food Chemistry, Vol. 55, No. 6, 2007, pp. 2142-2148. doi:10.1021/jf061714h

[20] K. Matsumoto, I. Ui, K. Satoh, T. Tobe, F. Ushio and K. Endo, "Evaluation of Oxidative Damage in the Liver of Selenium-Deficient Rats," Redox Report, Vol. 7, No. 5, 2002, pp. 351-354. doi:10.1179/135100002125000974

[21] J. K. Willcox, G. L. Catignani and L. J. Roberts, "Dietary Flavonoids Fail to Suppress F2-Isoprostane Formation in Vivo," Free Radical Biology \& Medicine, Vol. 34, No. 7, 2003, pp. 795-799. doi:10.1016/S0891-5849(02)01425-9

[22] E. J. Lien, S. Ren, H. H. Bui and R. Wang, "Quantitative Structure-Activity Relationship Analysis of Phenolic Antioxidants," Free Radical Biology \& Medicine, Vol. 26, No. 3-4, 1999, pp. 285-294. doi:10.1016/S0891-5849(98)00190-7

[23] E. J. Choi, K. M. Chee and B. H. Lee, "Anti- and Prooxidant Effects of Chronic Quercetin Administration in Rats," European Journal of Pharmacology, Vol. 482, No. 1-3, 2003, pp. 281-285. doi:10.1016/j.ejphar.2003.09.067

[24] F. Lucesoli, M. Caligiuri, M. F. Roberti, J. C. Perazzo and C. G. Fraga, "Dose-Dependent Increase of Oxidative Damage in the testes of Rats Subjected to Acute Iron Overload," Archives of Biochemistry and Biophysics, Vol. 372, No. 1, 1999, pp. 37-43. doi:10.1006/abbi.1999.1476

[25] L. Miles, M. V. Miles, P. H. Tang, P. S. Horn, J. G. Quinlan, B. Wong, A. Wenisch and K. E. Bove, "Ubiquinol: A Potential Biomarker for Tissue Energy Requirements and Oxidative Stress," Clinica Chimica Acta, Vol. 360, No. 1-2, 2005, pp. 87-96. doi:10.1016/j.cccn.2005.04.009

[26] A. Galinier, A. Carriere, Y. Fernandez, A. M. Bessac, S. Caspar-Bauguil, B. Periquet, M. Comtat, J. P. Thouvenot and L. Casteilla, "Biological Validation of Coenzyme Q Redox State by HPLC-EC Measurement: Relationship between Coenzyme Q Redox State and Coenzyme Q Content in Rat Tissues," FEBS Letters, Vol. 578, No. 1-2, 2004, pp. 53-57. doi:10.1016/i.febslet.2004.10.067

[27] F. Navarro, P. Navas, J. R. Burgess, R. I. Bello, R. De Cabo, A. Arroyo and J. M. Villalba, "Vitamin E and Selenium Deficiency Induces Expression of the UbiquinoneDependent Antioxidant System at the Plasma Membrane," FASEB Journal, Vol. 12, No. 15, 1998, pp. 1665-1673.

[28] G. Lenaz, M. D’Aurelio, M. Merlo Pich, M. L. Genova, B. 
Ventura, C. Bovina, G. Formiggini and G. Parenti Castelli, "Mitochondrial Bioenergetics in Aging," Biochimica et Biophysica Acta (BBA)-Bioenergetics, Vol. 1459, No. 2-3, 2000, pp. 397-404. doi:10.1016/S0005-2728(00)00177-8

[29] L. Ernster, P. Forsmark and K. Nordenbrand, "The Mode of Action of Lipid-Soluble Antioxidants in Biological Membranes: Relationship between the Effects of Ubiquinol and Vitamin E as Inhibitors of Lipid Peroxidation in Submitochondrial Particles," Biofactors, Vol. 3, No. 4, 1992, pp. 241-248.

[30] K. E. Hill and R. F. Burk, "Effect of Selenium Deficiency and Vitamin E Deficiency on Glutathione Metabolism in Isolated Rat Hepatocytes," Journal of Biological Chemistry, Vol. 257, No. 18, 1982, pp. 10668-10672.

[31] J. Frank, T. Lundh, R. S. Parker, J. E. Swanson, B. Vessby and A. Kamal-Eldin, "Dietary (+)-Catechin and BHT Markedly Increase Alpha-Tocopherol Concentrations in Rats by a Tocopherol-Omega-Hydroxylase-Independent Mechanism," Journal of Nutrition, Vol. 133, No. 10, 2003, pp. 3195-3199.

[32] J. Frank, A. Budek, T. Lundh, R. S. Parker, J. E. Swanson, C. F. Lourenco, B. Gago, J. Laranjinha, B. Vessby and A. Kamal-Eldin, "Dietary Flavonoids with a Catechol Structure Increase Alpha-Tocopherol in Rats and Protect the Vitamin from Oxidation in Vitro," Journal of Lipid Research, Vol. 47, No. 12, 2006, pp. 2718-2725. doi:10.1194/jlr.M600291-JLR200

[33] L. Fremont, M. T. Gozzelino, M. P. Franchi and A. Linard, "Dietary Flavonoids Reduce Lipid Peroxidation in Rats Fed Polyunsaturated or Monounsaturated Fat Diets," Journal of Nutrition, Vol. 128, No. 9, 1998, pp. 14951502.

[34] F. Nanjo, M. Honda, K. Okushio, N. Matsumoto, F. Ishigaki, T. Ishigami and Y. Hara, "Effects of Dietary Tea Catechins on Alpha-Tocopherol Levels, Lipid Peroxidation, and Erythrocyte Deformability in Rats Fed on High Palm Oil and Perilla Oil Diets," Biological \& Pharmaceutical Bulletin, Vol. 16, No. 11, 1993, pp. 1156-1159. doi:10.1248/bpb.16.1156

[35] M. A. Colucci, C. J. Moody and G. D. Couch, "Natural and Synthetic Quinones and Their Reduction by the Quinone Reductase Enzyme NQO1: From Synthetic Organic Chemistry to Compounds with Anticancer Potential," Organic \& Biomolecular Chemistry, Vol. 6, No. 4, 2008, pp. 637-656. doi:10.1039/b715270a

[36] K. B. Storey, "Oxidative Stress: Animal Adaptations in Nature," Brazilian Journal of Medical and Biological Research, Vol. 29, No. 12, 1996, pp. 1715-1733.

[37] A. K. Jaiswal, "Regulation of Genes Encoding NAD(P)H: Quinone Oxidoreductases," Free Radical Biology \& Medicine, Vol. 29, No. 3-4, 2000, pp. 254-262. doi:10.1016/S0891-5849(00)00306-3

[38] Y. J. Moon, X. Wang and M. E. Morris. "Dietary Flavonoids: Effects on Xenobiotic and Carcinogen Metabolism," Toxicology In Vitro, Vol. 20, No. 2, 2006, pp. 187-210. doi:10.1016/j.tiv.2005.06.048

[39] A. D. Kinghorn, B. N. Su, D. S. Jang, L. C. Chang, D. Lee, J. Q. Gu, E. J. Carcache-Blanco, A. D. Pawlus, S. K.
Lee, E. J. Park, M. Cuendet, J. J. Gills, K. Bhat, H. S. Park, E. Mata-Greenwood, L. L. Song, M. Jang and J. M. Pezzuto, "Natural Inhibitors of Carcinogenesis," Planta Medica, Vol. 70, No. 8, 2004, pp. 691-705. doi:10.1055/s-2004-827198

[40] C. Chen, R. Yu, E. D. Owuor and A. N. Kong. "Activation of Antioxidant-Response Element (ARE), MitogenActivated Protein Kinases (MAPKs) and Caspases by Major Green Tea Polyphenol Components During Cell Survival and Death," Archives of Pharmacal Research, Vol. 23, No. 6, 2000, pp. 605-612. doi:10.1007/BF02975249

[41] H. Y. Chan, H. Wang, D. S. Tsang, Z. Y. Chen and L. K. Leung, "Screening of Chemopreventive Tea Polyphenols against PAH Genotoxicity in Breast Cancer Cells by a XRE-Luciferase Reporter Construct," Nutrition and Cancer, Vol. 46, No. 1, 2003, pp. 93-100. doi:10.1207/S15327914NC4601 12

[42] S. K. Katiyar, R. Agarwal, M. T. Zaim and H. Mukhtar, "Protection against $N$-Nitrosodiethylamine and Benzo[a] Pyrene-Induced Forestomach and Lung Tumorigenesis in A/J Mice by Green Tea," Carcinogenesis, Vol. 14, No. 5, 1993, pp. 849-855. doi:10.1093/carcin/14.5.849

[43] S. G. Khan, S. K. Katiyar, R. Agarwal and H. Mukhtar, "Enhancement of Antioxidant and Phase II Enzymes by Oral Feeding of Green Tea Polyphenols in Drinking Water to SKH-1 Hairless Mice: Possible Role in Cancer Chemoprevention," Cancer Research, Vol. 52, No. 14, 1992, pp. 4050-4052.

[44] L. Qi and C. Han, "Induction of NAD(P)H: Quinone Reductase by Anticarcinogenic Ingredients of Tea," Wei Sheng Yan Jiu, Vol. 27, No. 5, 1998, pp. 323-326.

[45] D. J. Morre, D. M. Morre, H. Sun, R. Cooper, J. Chang and E. M. Janle, "Tea Catechin Synergies in Inhibition of Cancer Cell Proliferation and of a Cancer Specific Cell Surface Oxidase (ECTO-NOX)," Pharmacology and Toxicology, Vol. 92, No. 5, 2003, pp. 234-241. doi:10.1034/j.1600-0773.2003.920506.x

[46] S. Itoh, S. Nagaoka and K. Mukai, "Kinetic Study of the Tocopherol Regeneration Reaction by Biological Hydroquinones in Micellar Solution," Journal of Physical Chemistry A, Vol. 112, No. 3, 2008, pp. 448-456. doi:10.1021/jp075894p

[47] J. Kaikkonen, K. Nyyssonen, A. Tomasi, A. Iannone, T. P. Tuomainen, E. Porkkala-Sarataho and J. T. Salonen, “Antioxidative Efficacy of Parallel and Combined Supplementation with Coenzyme Q10 and d-Alpha-Tocopherol in Mildly Hypercholesterolemic Subjects: A Randomized Placebo-Controlled Clinical Study," Free Radical Research, Vol. 33, No. 3, 2000, pp. 329-340. doi:10.1080/10715760000301501

[48] B. Zhou, L. M. Wu, L. Yang and Z. L. Liu, "Evidence for Alpha-Tocopherol Regeneration Reaction of Green Tea Polyphenols in SDS Micelles," Free Radical Biology \& Medicine, Vol. 38, No. 1, 2005, pp. 78-84. doi:10.1016/j.freeradbiomed.2004.09.023

[49] F. Dai, W. F. Chen and B. Zhou, "Antioxidant Synergism of Green Tea Polyphenols with Alpha-Tocopherol and LAscorbic Acid in SDS Micelles," Biochimie, Vol. 90, No. 
10, 2008, pp. 1499-1505. doi:10.1016/j.biochi.2008.05.007

[50] Y. Kadoma, M. Ishihara, N. Okada and S. Fujisawa, "Free Radical Interaction between Vitamin E (Alpha-, Beta-, Gamma- and Delta-Tocopherol), Ascorbate and Flavonoids," In Vivo, Vol. 20, No. 6B, 2006, pp. 823-827.

[51] Q. Y. Zhu, Y. Huang, D. Tsang and Z. Y. Chen, "Regeneration of Alpha-Tocopherol in Human Low-Density Lipoprotein by Green Tea Catechin," Journal of Agricultural and Food Chemistry, Vol. 47, No. 5, 1999, pp. 2020-2025. doi:10.1021/jf9809941

[52] S. V. Jovanovic and M. G. Simic, "Antioxidants in Nutrition," Annals of the New York Academy of Sciences, Vol. 899, 2000, pp. 326-334.

doi:10.1111/j.1749-6632.2000.tb06197.x
[53] K. Furuno, T. Akasako and N. Sugihara, "The Contribution of the Pyrogallol Moiety to the Superoxide Radical Scavenging Activity of Flavonoids," Biological \& Pharmaceutical Bulletin, Vol. 25, No. 1, 2002, pp. 19-23. doi:10.1248/bpb.25.19

[54] M. Muzolf-Panek, A. Gliszczynska-Swiglo, L. de Haan, J. M. Aarts, H. Szymusiak, J. M. Vervoort, B. Tyrakowska and I. M. Rietjens, "Role of Catechin Quinones in the Induction of EpRE-Mediated Gene Expression," Chemical Research in Toxicology, Vol. 21, No. 12, 2008, pp. 2352-2360. doi:10.1021/tx8001498

[55] J. George, "Elevated Serum Beta-Glucuronidase Reflects Hepatic Lysosomal Fragility Following Toxic Liver Injury in Rats," Biochemistry and Cell Biology, Vol. 86, No. 3, 2008, pp. 235-243. doi:10.1139/O08-038

\section{Abbreviations Used}

ANOVA: analysis of variance;

bw: body weight;

CoQ: coenzyme Q;

Hox: high oxidative stress;

Lox: low oxidative stress;

NQO1, NAD(P)H: quinone oxidoreductase-1;

ROS: reactive oxygen species. 